\title{
LA FAMILIA EN EL CUIDADO A LA SALUD SEXUAL DE LOS ADOLESCENTES
}

\author{
The family in caring for sexual health of adolescents
}

\section{EPISTEMUS}

ISSN: 2007-8196 (electrónico)

ISSN: 2007-4530 (impresa)

Mayra Jeniffer Ramírez González ${ }^{1}$

Alicia Álvarez Aguirre ${ }^{2}$

Mayra Itzel Huerta Baltazar ${ }^{3}$

María de Jesús Ruíz Recéndiz ${ }^{4}$

María Mercedez Moreno González ${ }^{5}$

Recibido: 20 de septiembre de 2018 ,

Aceptado: 30 de noviembre de 2018

Autor de Correspondencia:

Mayra Jeniffer Ramírez González

Correo:mj.ramirezgonzalez@ugto.mx

\section{Resumen}

La familia representa un sistema social y natural, está conformada por una red de relaciones que dan respuesta a las necesidades biológicas y psicológicas inherentes a la supervivencia humana; brinda protección, estabilidad, valores y pertenencia, promueve el desarrollo y adaptación de los adolescentes, además de intervenir cuando surgen problemas. La adolescencia, es una etapa de transición donde ocurren diversos cambios biopsicosociales que tienen como objetivo la formación de una identidad, para ello dependen de su familia pues a través de ella logran adquirir toda una serie de competencias importantes que pueden ayudarles a una transición satisfactoria de la infancia a la edad adulta. Ante esto, los profesionales de enfermería, desarrollan competencias para promover la cooperación, el apoyo mutuo y la coordinación en las familias para propiciar formas de adaptación a las diferentes situaciones que vive la familia.

Palabras clave: Familia, salud sexual, adolescentes

\section{Abstract}

The family represents a social and natural system, is formed by a network of relationships that respond to the biological and psychological needs inherent in human survival; provides protection, stability, values and belonging, promotes the development and adaptation of adolescents, in addition to intervening when problems arise. Adolescence is a stage of transition where various biopsychosocial changes that aim to form an identity occur, for this they depend on their family because through it they manage to acquire a series of important competences that can help them to make a successful transition from childhood to adulthood. Given this, nursing professionals develop skills to promote cooperation, mutual support and coordination in families to promote ways of adapting to the different situations experienced by the family.

Keywords: family, sexual health, adolescents

\footnotetext{
1 División de Ciencias de la Salud e Ingenierías, Campus Celaya-Salvatierra, Universidad de Guanajuato. México / Correo: mj.ramirezgonzalez@ugto.mx 2 Departamento de Enfermería Clínica, División de Ciencias de la Salud e Ingenierías, Campus Celaya-Salvatierra, Universidad de Guanajuato. México / Correo:alicia.alvarez@ugto.mx

3 Facultad de Enfermería de la Universidad Michoacana de San Nicolás De Hidalgo. México / Correo: Efetakumi@gmail.com

4 Facultad de Enfermería de la Universidad Michoacana de San Nicolás De Hidalgo. México / Correo: Violetarr05@gmail.com

5 Departamento de Enfermería Clínica, División de Ciencias de la Salud e Ingenierías, Campus Celaya-Salvatierra de la Universidad de Guanajuato. México / Correo:ma.moreno@ugto.mx 5
} 


\section{INTRODUCCIÓN}

La familia representa una parte fundamental en la formación de los hijos; brinda protección, estabilidad, valores, pertenencia, herramientas para establecer relaciones con otros individuos de la familia y la sociedad. También es motor para la prevención de diferentes conductas sociales y de salud. [1]

Los problemas de salud en la familia son aquellos que involucran al total de la familia o a dos o más de sus miembros por ejemplo los problemas de la crianza de los hijos, la comunicación, los conflictos de rol-afectivos, las prácticas deficientes de las dimensiones del estilo de vida tales como actividad física, nutrición, manejo del estrés , entre otros. [2]

En el contexto familiar, la comunicación asertiva favorece el bienestar de los integrantes, en el caso de las familias con hijos adolescentes hace que sea menos probable que se involucren en conductas de riesgo, tales como accidentes de tránsito relacionados con el consumo de alcohol, VIH, suicidio, la violencia interpersonal, consideradas de mayor prevalencia en este grupo y que llevan al adolescente a la muerte. [3]

Los problemas de salud pueden afectar la capacidad de los adolescentes para crecer y desarrollarse plenamente, las conductas como el consumo de alcohol o tabaco, la falta de actividad física, las relaciones sexuales sin protección y/o la exposición a la violencia, pueden poner en peligro no solo su salud actual, sino también la de años posteriores e incluso la salud de sus futuros hijos. [3-5]

En relación a la conducta sexual de los adolescentes, los padres tienen una determinante influencia en sus hijos, los estudios reportan que la comunicación acerca del cuidado de la salud sexual se asocia positivamente con la conducta sexual segura, retraso de inicio de vida sexual activa e incremento de uso de condón, disminución de embarazos precoces, abortos, ITS y VIH. [6-7]
Sin embargo, pocos padres hablan sobre este tema con sus hijos, generalmente los padres no se sienten seguros o capaces de llevar a cabo una comunicación sobre el cuidado de la salud sexual, [8-9] lo anterior, debido a ciertos factores que influyen en la comunicación entre padres e hijos como la capacidad de iniciar y mantener el diálogo, el conocimiento de los padres sobre salud sexual y reproductiva, así como la cultura, creencias y valores que caracterizan el medio ambiente familiar.[10-11] No obstante los estudios reportan que los adolescentes expresan que prefieren hablar con sus padres de sexualidad [8-12] y que aquellos adolescentes que tienen una comunicación efectiva con sus padres presentan mayor autoestima y motivación, ayudándolos a involucrarse en el cuidado de su salud sexual de forma responsable y segura. [13]

Por lo antes descrito el objetivo de este artículo es analizar el rol de la familia en el cuidado de la salud sexual de los adolescentes desde la perspectiva de enfermería familiar, a fin de generar las bases de una cultura de comunicación entre padres e hijos sobre el cuidado de la salud sexual.

\section{DESARROLLO}

\section{Familia y adolescencia}

La familia es un grupo social que aparece con el hombre y evoluciona de acuerdo a su historia, en el que se mantienen valores fundamentales que sustentan sus integrantes debido a que estos han logrado un cierto nivel de consenso. [1]

Además, la familia involucra a dos o más individuos unidos por lazos de matrimonio, sangre o adopción, que dependen entre sí para apoyos emocionales, físicos y económicos, además de cumplir con las funciones relacionadas a asuntos sociales y de salud. [14]

A la luz de las definiciones anteriores la familia es un conjunto dinámico y potencial de símbolos, creencias, actividades, funciones y relaciones interpersonales que se desarrollan e integran en el contexto primario más importante del desarrollo humano. [22]

Para los seres humanos, la familia representa el grupo más importante en relación con el desarrollo físico, psicológico, social y espiritual. Para varios la familia es un conjunto de personas en el que se experimenta amor y odio, y dentro del cual se disfruta de las satisfacciones más profundas y se padecen las desilusiones más dolorosas. Los integrantes de la familia se vinculan por lazos intensos y duraderos derivados de experiencias pasadas, roles sociales, apoyo y necesidades. [23]

La familia cumple con cuatro funciones fundamentales respecto a sus hijos: la primera es asegurar su supervivencia y sano crecimiento físico, 


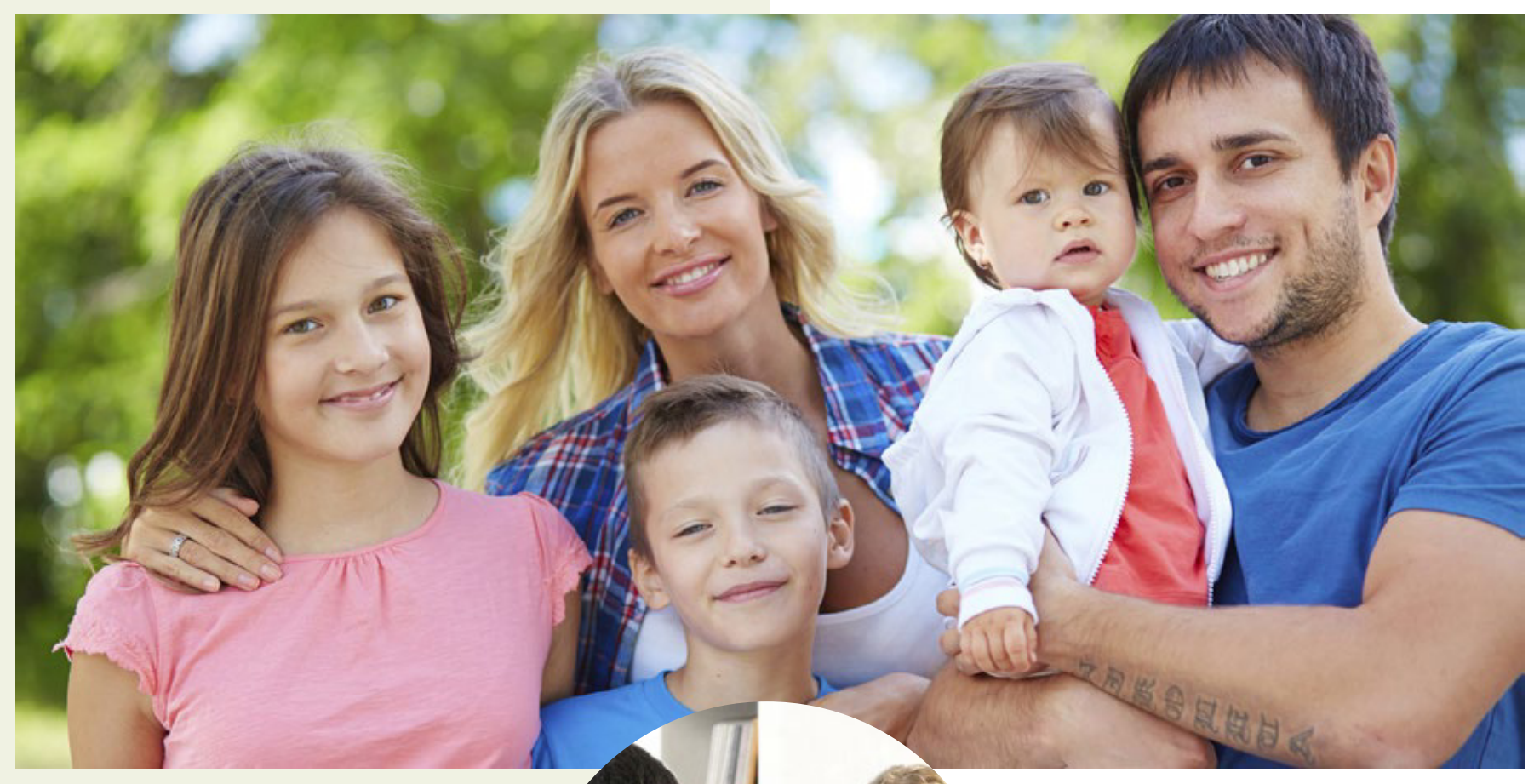

la segunda, es brindarles afecto y apoyo pues sin esto el desarrollo psicológico sano no es posible, el tercero implica la capacidad para relacionarse adecuadamente con su entorno físico y social, el cuarto corresponde a la educación en familia que implica la toma de decisiones con respecto a la apertura hacia otros contextos educativos y sociales. Para que estas funciones sean llevadas a cabo se debe dedicar mayor tiempo a la relación con los hijos, además, se requiere de una alianza efectiva basada en interacciones habituales y relajadas en diversos contextos y con distintas finalidades como son los cuidados físicos y sexuales. [1]

Así pues, la familia implica cierto nivel de riesgo en uno o varios de los integrantes, trátese del padre, madre o hijos, de modo que, la familia juega un papel fundamental en la protección de los miembros, principalmente en la de los hijos adolescentes, pues es en la adolescencia donde ocurren diversos cambios biopsicosociales que tienen como objetivo la formación de una identidad, y es aquí donde los adolescentes pueden exponerse a riegos de salud. [4-14]

De acuerdo a la Organización Mundial de la Salud (OMS), la adolescencia es el periodo de crecimiento y desarrollo humano que se produce después de la niñez y antes de la edad adulta. La adolescencia, es una de las etapas de transición más importantes en la vida del ser humano, que se caracteriza por un ritmo acelerado de crecimiento y de cambios. Esta fase de crecimiento y desarrollo viene condicionada por diversos procesos biológicos. [3]

y emocionalmente para enfrentar el proceso de la reproducción, tienden a iniciar una vida sexual activa, cimentada en relaciones pasajeras, carentes de amor, donde el continuo cambio de pareja es habitual y los lleva a conductas riesgosas, que pueden traer consigo un embarazo no deseado o bien sufrir una ITS incluido el VIH.

En definitiva, los adolescentes dependen de su familia para adquirir toda una serie de competencias importantes que pueden ayudarles a hacer una transición satisfactoria de la infancia a la edad adulta. De modo que, este proceso representa para los padres una gran oportunidad para influir en sus hijos.

\section{LA SALUD SEXUAL EN LA FAMILIA CON HIJOS ADOLESCENTES}

La sexualidad humana es una dimensión fundamental basada en el género, orientación sexual, erotismo, vínculo emocional, amor y reproducción. Es expresada en diversas maneras como deseos, pensamientos, fantasías, creencias, actitudes, valores, practicas, actividades y relaciones. La sexualidad es el producto final del intercambio de factores 
biológicos, psicológicos, socioeconómicos, culturales, éticos, religiosos y espirituales; es practicada y expresada por la persona en su ser, sus sentimos y pensamientos. [16]

Durante la adolescencia surgen presiones para consumir alcohol, tabaco u otras drogas y para empezar a tener relaciones sexuales, a edades cada vez más tempranas, lo que entraña en ellos un elevado riesgo de traumatismos, tanto intencionados como accidentales, embarazos no deseados e ITS entre ellas el VIH. [3]

La sexualidad en la adolescencia se ha convertido en un problema de Salud Pública ya que estadísticamente se estima que a nivel nacional solo dos de cada 10 adolescentes que inician vida sexual se protegen con algún método anticonceptivo, poniendo en riesgo su vida y salud. [9] Dado que, un adolescente no es plenamente capaz de comprender la relación entre una conducta y sus consecuencias, ni de percibir el grado de control que puede tener respecto a la toma de decisiones relacionadas con la salud, por ejemplo en su comportamiento sexual.

Los compañeros del colegio y el contexto sociocultural asumen un papel fundamental en el comportamiento de los adolescentes; sin embargo quien influye directamente en ellos es la familia, ya que es donde los hijos retoman lo que han aprendido para relacionarse con su pareja. En este sentido la familia puede prevenir conductas que ponen en riesgo la vida y salud de los adolescentes. [11-17]

Los adolescentes presentan elevadas necesidades de salud, debido a que la mayoría del tiempo se involucra en comportamientos de riesgo, como el consumo de alcohol y prácticas sexuales de riesgo; todo esto debido a un juicio deteriorado, baja autoestima, conflictos en las relaciones interpersonales, entre otras. Por ello, es necesario implementar intervenciones preventivas para disminuir los patrones de riesgo, puesto que estudios previos han demostrado que los comportamientos de riesgo que comienzan en la adolescencia persisten en la edad adulta. [18]

La evidencia muestra que la familia influye en el comportamiento sexual de riesgo de los adolescentes, pues representan un factor directo del conocimiento y actitudes de sus hijos. Por tal motivo las futuras intervenciones deben estar enfocadas a los padres y deben ser diseñadas para proporcionar conocimiento y desarrollar habilidades necesarias acerca de la comunicación sobre tópicos de sexualidad. [13] Aunado a que las intervenciones que toman en cuenta la participación de los padres y adolescentes, traen consigo mejores resultados en comparación con aquellos programas que únicamente son dirigidos a los adolescentes. [11]

\section{COMUNICACIÓN ENTRE PADRES E HIJOS SOBRE LA SALUD SEXUAL}

La comunicación es un proceso innato del hombre, una necesidad básica, a través de la comunicación se conoce el mundo, se organizan pensamientos, se expresan sentimientos y se comprenden las ideas de los demás. La

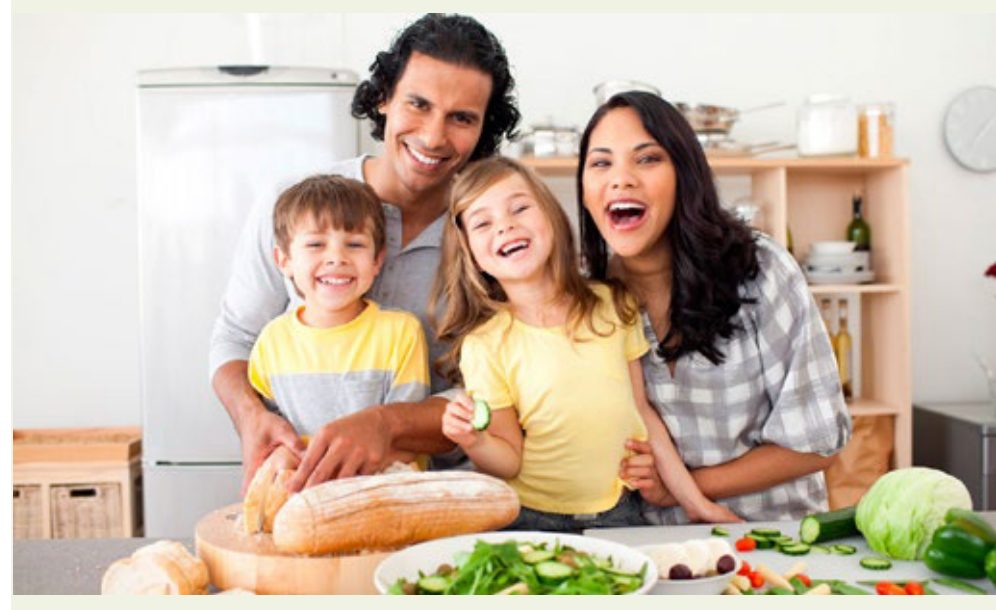

comunicación es crucial para una buena formación, se expresan dudas y se comparte información, el dialogo promueve la toma de decisiones sanas y disminuye riesgos. [11-17]

Bandura señala que mediante la comunicación se crean habilidades protectoras a los riesgos, que de cierto modo ejercen un control que beneficia el autocuidado; en el manejo de situaciones sexuales de riesgo. Las habilidades protectoras están ligadas a la comunicación clara y efectiva entre padres e hijos. [19]

Por su parte Satir, refiere que el proceso de la comunicación interpersonal que existe en la familia, incluye quien se comunica con quién, cómo, cuándo y el estilo que adopta la comunicación, dando como resultado cinco estilos de comunicación propios de la familia, el primero; culpar o criticar al otro, el segundo; racionalizar, para justificar la manera de actuar y/o pensar que las personas tratan de hallar explicaciones, tercero; en este apartado uno o varios miembros de la familia evita tratar temas originados en la comunicación, cuarto; complacer a otro, generalmente podría resultar satisfactorio, sin embargo, también es un problema para la comunicación, pues impide la discusión de los acontecimientos y quinto, la congruencia o asertividad, que permite a los integrantes de la familia ponerse en el lugar del otro. [1]

Tanto la comunicación como la supervisión de los padres hacia sus hijos, tiene un impacto importante en el desarrollo de comportamientos de riesgo, estudios han demostrado que el aumento de la comunicación entre padres y adolescentes sobre tópicos de sexualidad influye al retrasar el debut sexual, asimismo aumenta la probabilidad de usar métodos anticonceptivos y rechazar relaciones sexuales no deseadas. Además, logra que los hijos se sientan confortables, aceptados y aprobados por sus padres al comunicarse con ellos. [17-20]

Se debe considerar que una de las preocupaciones de los padres al entablar este tipo de conversaciones con sus hijos es el miedo de no poder ser capaces a responder ciertas preguntas relacionadas con la salud sexual. Para combatir este miedo, se debe abordar la autoeficacia de los padres, para poder entablar una comunicación con sus 


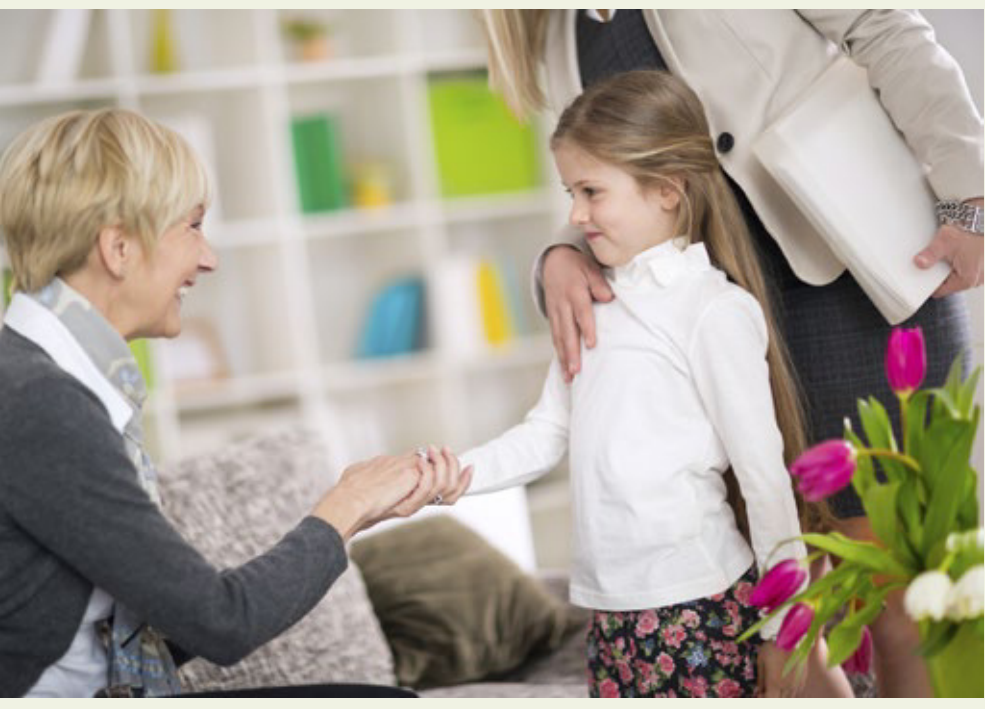

hijos. [20] La autoeficacia se basa en el juicio que posee un individuo sobre sus capacidades para organizar y ejecutar cursos de acción. [22] Es decir, la capacidad que tienen los padres para iniciar y mantener el diálogo, el conocimiento de los padres sobre salud sexual y reproductiva, así como la cultura, creencias y valores que caracterizan el medio ambiente familiar para hablar sobre tópicos de sexualidad. [10-11]

\section{IMPLICACIONES PARA LA INVESTIGACIÓN EN ENFERMERÍA FAMILIAR}

La profesión de enfermería incluye una atención autónoma y en colaboración dirigida a personas de todas las edades, familias, grupos y comunidades, enfermos o no. Comprende la promoción de la salud, la prevención de enfermedades y atención a enfermos, discapacitados y personas en situación terminal. [21]

En relación al trabajo con familias, el profesional de enfermería debe tener una preparación rigurosa y desarrollar un sentimiento de unidad para promover la cooperación, el apoyo mutuo y la coordinación; propiciar formas de adaptación a las diferentes situaciones a las que se deben enfrentar; ver a las familias como cuidadoras de sus propios miembros, e identificar el hogar como el lugar en que viven el paciente y sus familiares. [22]

El cuidar a la familia es un reto profesional, dado que, la enfermera sale de los esquemas del cuidado, trátese de hospitales o centros de salud, donde la situación familiar se vislumbra escasamente a plena luz.

La investigación para la prevención de diversas ITS incluido el VIH en adolescentes crea desafíos debido a las consideraciones éticas y morales asociadas con la etapa de desarrollo de la población. Sin embargo, la participación de los padres desde el inicio hasta el término de la intervención es fundamental para la prevención del $\mathrm{VIH}$. [23]

En la actualidad, gran parte de los padres se preocupan por el desarrollo de los hijos en las tareas escolares, deportivas y sociales, resulta importante destacar que su función fundamental es crear relaciones armoniosas y estrechas con ellos. Es importante que los padres mantengan una comunicación sobre la salud sexual con sus hijos, para ello deben tener conocimiento, comprensión y motivación; la anatomía y fisiología de la reproducción humana, las ITS y la concepción así como los factores que aumentan el riesgo de ITS y la abstinencia, son algunos de los temas que pueden abordar los padres con sus hijos. [25]

En lo que respecta al desarrollo social, esta etapa presenta una disminución de la influencia familiar, los valores se hacen menos importantes a medida que el adolescente se siente más cómodo con sus propios principios e identidad. Las amistades se hacen menos y más selectivas, incluso, el adolescente vuelve a acercarse a la familia, aumentado gradualmente la intimidad con sus padres si ha existido una relación positiva con ellos durante los años previos. [26]

Por ello, se debe trabajar más en intervenciones entre padres e hijos; los investigadores deben abarcar factores de protección que beneficien el funcionamiento familiar en materia de comunicación sobre tópicos de sexualidad. Para ello, se requiere de un mayor número de investigaciones que favorezcan la toma de decisiones de los hijos, especialmente cuando se trata de alguna implicación en su vida y salud. [14]

Es importante señalar que las enfermeras deben familiarizarse con las intervenciones para obtener resultados innovadores, dado que juegan un papel fundamental en la familia, pues son proactivas en el desarrollo de políticas que promueven la salud de los integrantes, su funcionamiento, procesos y relaciones a lo largo de la vida. Motivo por el cual, el profesional de enfermería debe manejar eficazmente aquellos procesos familiares que modifican los factores de riesgo y promuevan los protectores. [10-14]

\section{CONCLUSIONES}

Los adolescentes dependen de la familia, comunidad, escuela y servicios de salud para conseguir competencias importantes que pueden ayudarles a hacer frente a las presiones que experimentan y lograr una adecuada transición de la infancia a la edad adulta. La familia posee gran compromiso para promover el desarrollo y la adaptación de los adolescentes y de intervenir eficazmente cuando surjan problemas.

Si el adolescente crece dentro de una familia donde exista buena comunicación, lo ayuda a sentirse valioso y querido, promueve un desarrollo pleno y saludable ante conductas como el consumo de alcohol o tabaco, la falta de actividad física y las relaciones sexuales sin protección, que pueden poner en peligro su salud.

Una comunicación efectiva sobre tópicos de sexualidad con los hijos; es fundamental para su formación, pues la adolescencia se caracteriza por el incremento de la experimentación sexual, 


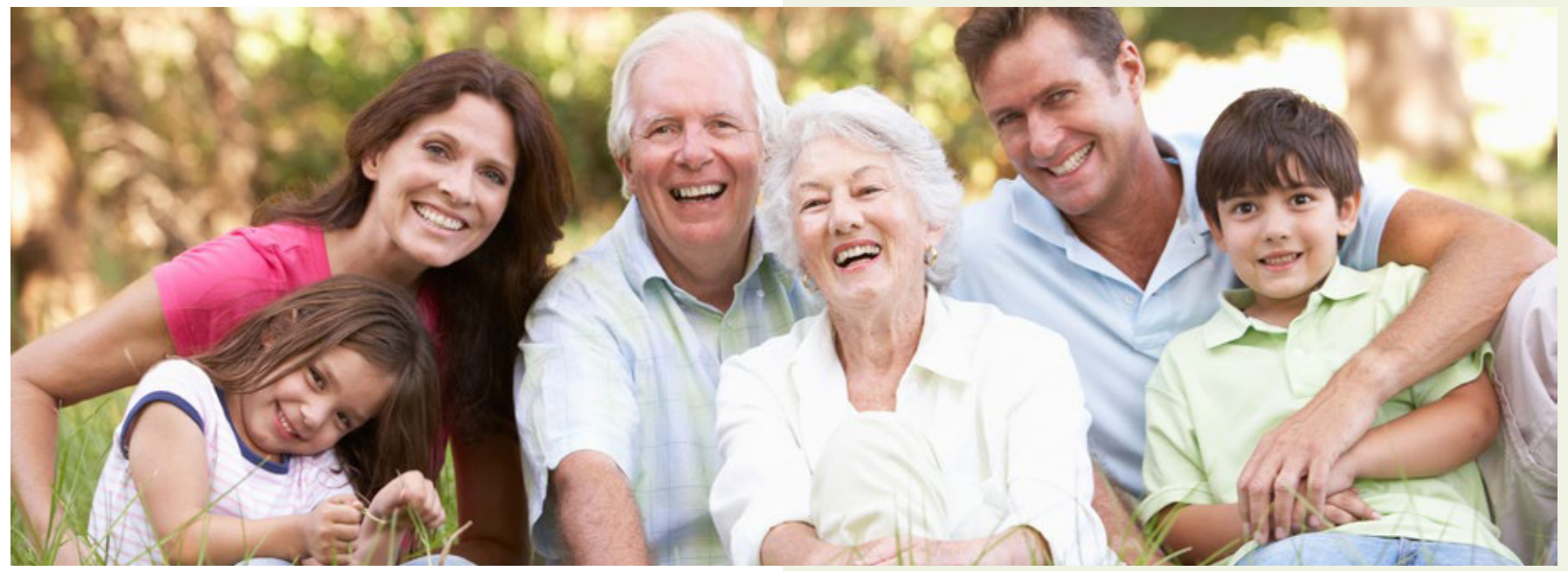

Así pues, podemos concluir que las habilidades protectoras son propias de los padres, son predictores importantes y además desempeñan un papel fundamental para el conocimiento y actitudes de sus hijos ante comportamientos sexuales.

\section{BIBILIOGRAFÍA}

[1] A. Valdés, "Familia y desarrollo. Intervenciones en terapia familiar," 1era ed. México: El manual moderno; 2013.

[2] M. Friedman, "Family nursing. Theory and assessment. Introduction to the family, The United States of America, Appleton Century Crofts," pp. 338, 1981.

[3] Organización Mundial de la Salud. Adolescentes: riesgos para la salud y soluciones, nota descriptiva: OMS; 2016.

[4] J. Parra and R. Pérez, "Comportamiento sexual en adolescentes y su relación con variables biosociales," Perinatología y Reproducción Humana., vol. 24, no. 1, pp. 7-19, 2010.

[5] M. A. Méndez, M. A. Mejía, M. C. Ávila, "Actitud de los docentes de licenciatura en educación hacia el VIH," MeDULA., vol. 19, no. 2, pp. 1, 2010.

[6] M. I. Huerta, "Factores predictores de conducta sexual de riesgo para VIH/SIDA en jóvenes universitarios de Morelia, México," tesis de Doctorado, Universidad de Guanajuato, Celaya Guanajuato, 2015.

[7] M. Pullupaxi, "La carencia afectiva como factor determinante en el inicio precoz de las relaciones sexuales en las adolescentes de 13 a 18 años de edad, estudiantes del colegio técnico Agoyán del cantón baños. Universidad técnica de Ambato," 2011. [Online]. Available:: http:// repositorio.uta.edu.ec/bitstream/123456789/3956/1/ TESIS\%20FINAL.pdf

[8] L. Castillo, et al, "Autoeficacia de padres para hablar con sus hijos acerca de sexo." Ciencia UANL., vol. 18, no. 73, pp. 45-46, 2015.

[9] S. Jiménez, P. Andrade and D. Betancourt, "Habilidades de comunicación en la conducta sexual de riesgo en jóvenes," Revista Intercontinental de Psicología y Educación., vol. 9, no. 002, pp. 147-162, 2007.

[10] G. Prado., et al, "Effects of a Family Intervention in Reducing HIV Risk Behaviors Among High-Risk Hispanic Adolescents," Online firts., vol. 166, no. 2, pp. 7, 2012

[11] B. Wang., et al, "The impact of parent involvement in an effective adolescent risk reduction intervention on sexual risk communication and adolescent outcomes," AIDS Educ Prev., vol. 26, no. 6, pp. 1-4, Dic. 2014.

[12] J. Mosquera and J. Mateus, "Conocimientos, actitudes y prácticas sobre métodos de planificación familiar, VIH/ SIDA y el uso de los medios de comunicación en jóvenes," Colombia médica., vol. 34, no. 4, pp. 206-212, 2003.

[13] M. Bangpan, and D. Operario, "Understanding the role of family on sexual-risk decisions of young women: A systematic review," AIDS Care., vol. 24, pp. 2-11, Julio. 2012.

[14] R. W. Eustace, "A discussion of HIV/AIDS family interventions: implications for family-focused nursing practice." Journal of Advanced Nursing., vol. 69, no. 7, pp. 2-4, 2012.

[15] C. Kuoa., et.al, "Developing family interventions for adolescent HIV prevention in South Africa," Aids Care., vol. 28, no. 1, pp. 1-4, 2016.

[16] M. Fallas, et al, "Justicia social para una vivencia afectiva y sexual en Costa Rica," Revista Ensayos Pedagógicos., vol. 11, no. 1, pp. 5, 2016.

[17] R. Sánchez and S. Robles, “Apoyo, supervisión y comunicación con padres y su relación con el comportamiento sexual de jóvenes en conflicto con la ley," Acta de investigación psicológica., vol. 4, no. 1, pp. 1339, 1406-1408, 2014.

[18] M. McKay, et al., "The Development and Implementation of Theory-Driven Programs Capable of Addressing PovertyImpacted Children's Health, Mental Health, and Prevention Needs: CHAMP and CHAMPp, Evidence-Informed, FamilyBased Interventions to Address HIV Risk and Care," Journal of Clinical Child \& Adolescent Psychology., vol. 43, pp. 2-4,11, 2014

[19] Bandura A, "Social cognitive theory: an agentic perspective," Asian journal of social psychology., vol. 2, p. 8-12, 1999. [Online]. Available: https://www.uky.edu/ eushe2/Bandura/ Bandura1999AJSP.pdf

[20] V. N. Carmon, et al, "Expectations and self-efficacy of African American parents who discuss sexuality with their adolescent sons: an intervention study," Public Health Nursing., vol. 31, no. 3, pp. 1,2,7, 2013.

[21] Organización Mundial de la Salud. Enfermería, nota descriptiva: OMS; 2018.

[22] M. Friedman. "Family nursing. Theory and assessment. Introduction to the family," The United States of America, Appleton Century Crofts, 338, 1981.

[23] J. Vig., et al, "Involving parents from the start: Formative evaluation for a large RCT with Botswana Junior Secondary School students," HHS Public Access., vol. 15, no. 1, pp. 2-13, Mar. 2016.

[24] M. Smith and P. Liehr. "Middle range theory for nursing."

[25] G. M. Bulechek, et al, "Clasificación de Intervenciones de Enfermería (NIC)," 6º ed. España: Elsevier, 2014.

[26] V. Gaete. "Desarrollo biopsicosocial del adolescente," Revista Chilena de Pediatría., vol. 86, no. 6, pp. 436-443, Nov. 2015. 